\title{
A REMOTE LABORATORY EXPERIMENTATION NETWORK
}

\author{
Christian Schmid $^{* 1}$, Tor Ivar Eikaas ${ }^{2}$, Bjarne Foss ${ }^{3}$, Denis Gillet ${ }^{4}$ \\ * Corresponding Author \\ ${ }^{1}$ Lehrstuhl für Elektrische Steuerung und Regelung \\ Ruhr-Universität Bochum IC-3/141 \\ D-44780 Bochum, Germany \\ Phone: +49234 32 24093, Fax: +492343204093 \\ E-mail:cs@esr.ruhr-uni-bochum.de \\ ${ }^{2}$ Cyberlab.Org AS, Trondheim, Norway \\ ${ }^{3}$ Norwegian University of Science and Technology, Trondheim, Norway \\ ${ }^{4}$ Swiss Federal Institute of Technology, Lausanne, Switzerland
}

\begin{abstract}
This contribution presents a remote laboratory project with a new business model that aims at bringing physical experimentation back into the learning arena, where remotely operable laboratory experiments used in advanced education and training schemes are made available to a global market. This is done via the Internet using a set of e-commerce and advanced information and communication technology solutions. The project will add online remote experimentation to distance learning techniques. This creates new opportunities for distance learning, in particular within the engineering disciplines where hands-on experience is regarded as essential to acquire knowledge and reinforce professional skills. We will assemble and integrate remote online experimentation with other multimedia learning resources like virtual reality and simulations, and develop course modules utilising the complementary learning effects of physical experiments.
\end{abstract}

Keywords: Control education, Laboratory education, Networks, Control technology, e-Commerce

\section{INTRODUCTION}

During the last decades there has been a decline in the use of physical experiments in engineering education. Learning is accomplished through a diversified set of activities like lectures, paper and pencil exercises, simulators, pilot experiments, discussions, and report writing. It is widely recognised that a mixture of theoretical and practical exercises is vital for efficient learning, and physical experiments constitute an important and complementary ingredient in learning, especially in the engineering and physics domain (Antsaklis et al., 1999; Kheir et al., 1996). Engineering students need to put the theory they learn during lectures into practice, which includes experimentation on real processes. Even quite rigorous simulators do not fully demonstrate the many practical problems related to operation of realworld equipment, and the trend towards the increased use of simulators in learning programs can only partially compensate for the decline in the use of physical experiments. There are several reasons for the decline described above. One important reason is the fact that physical experiments are costly to develop, maintain and operate. Another reason has been a strong belief that simulators can fully replace physical experiments. The ability to simulate a process is clearly very helpful for finding solutions to may real-world problems. The knowledge about modelling and simulation are therefore important skills for an engineer. Nevertheless, it is the strong believe of the authors that physical experiments con- 
stitute an important ingredient in order to carry out real hands-on learning activities.

The project is based on the idea that although a physical process cannot easily be moved, it can be made available for on-line remote experimentation via the Internet. The providers of laboratory facilities will be paid for the use of their facilities, while the customers are relieved of the costs involved in building and maintaining the laboratory equipment. This also enables a much more efficient use of laboratory facilities, since laboratory equipment in universities is at present used for only a fraction of the academic year. A key aspect of the project is the hypothesis that a commercial company is needed for providing the dedicated services required by this new remote experimentation paradigm. As a matter of fact, classical universities are hardly organised to offer such Internet services.

The remote experimentation providers and users, as well as the service provider, together form a network of partners. During 2000 and 2001 a trial network obtains funding from the European Commission through the IST programme to conduct the ReLAX "Remote LAboratory eXperimentation trial" project (ReLAX, 2001), which includes participants from different countries. It should be noted that there is an intense interest from the EU towards innovative Internet-based education concepts. The participants of the ReLAX project are from Norway (Cyberlab.Org AS, Prediktor AS, Norwegian University of Science and Technology (NTNU)), Germany (RuhrUniversität Bochum (RUB)) and from Switzerland (Swiss Federal Institute of Technology (EPFL)).

\section{ORGANISATIONAL STRUCTURE OF THE CYBERLAB NETWORK}

The main objective of ReLAX is the trial of a new business model based on an Experiment Service
Provider offering services utilising remote experimentation.

The idea behind the business model is to organise the network of laboratory experiments through an independent organising company, the Experiment Service Provider. The Experiment Service Provider offers a set of laboratory experiments to Customers (universities, individuals and industrial companies) on a global basis through a lab-portal and a Webbased trading system, see Fig. 1. The experiments are high quality experiments offered by educational institutions (Providers).

The Experiment Service Provider is responsible for the commercial aspects like quality assurance, marketing, booking system for assigning experiments and time slots, access control, brokerage, invoicing, secure electronic payment, handling of disputes, SW services, etc. By joining this network, the experiment Providers can benefit from the services from the Experiment Service Provider and at the same time benefit from an increased income through the exploitation of own laboratory resources. The economic and educational benefits for the Customers is the access to high-quality experiments bringing added value for own education, training or services.

A new company Cyberlab.Org AS will act as the Experiment Service Provider in the network. An important objective is to provide easy integration in the network of any HW and SW system used by the Provider.

The Service Provider will specify key aspects of how the user should be able to interact with the process, and will assist the Provider in connecting the experiment to the Internet, if the Provider requires such assistance. However, technical specifications

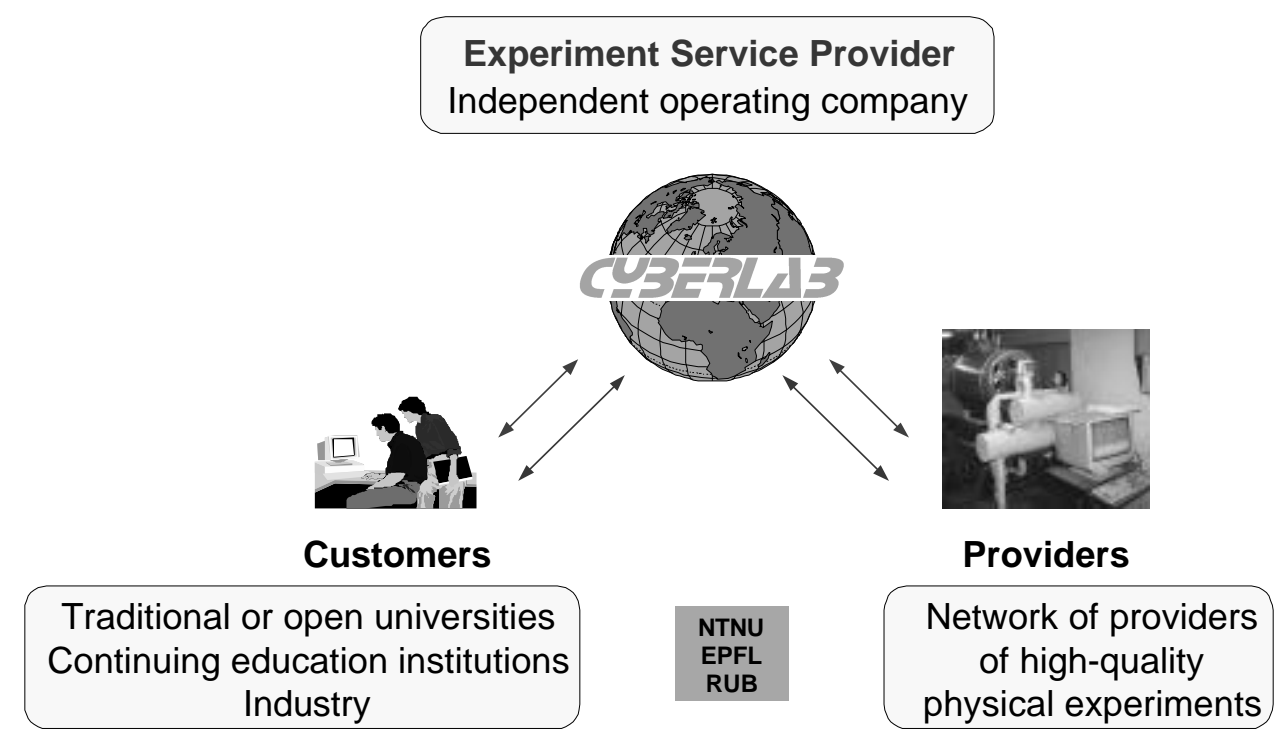

Fig. 1. Organisation of the remote laboratory experimentation network. 
will be independent of the actual hardware and software the Provider uses.

The Provider will connect a laboratory experiment to the Internet through a Web service, and the user interacts with the experiment through a Web browser. The information received from the experiment will typically include time series plots, video and audio information. When the experiment is completed, it should also be possible to obtain comprehensive documentation of the experiment by downloading files consisting of time series of experimental results. Exactly which variables the user should be able to manipulate remotely, as well as when, how and how much the variables can be manipulated, will depend on the design of each experiment.

Each Provider may concentrate their effort on maintaining a few high-quality experiments for remote on-line experimentation, while having access to many other remote laboratories from other Providers. Further, other users, without their own laboratory facilities, may purchase access to remote laboratories. This latter category of users will include universities that have given up on practical experiments due to high costs, open universities, commercial organisations offering continuing education courses, and in-house training departments in engineering and production companies. There are a number of technological and practical issues that need to be resolved in order to achieve a similar level of closeness when running a remote experiment than when running the same experiment locally.

Within the network, an external user group will be established and designed to obtain the right interaction with possible future customers from industry and open learning institutions. The world leading distributor of distance education, Open University (UK) is invited to participate together with FernUniversität Hagen, Germany, a leading provider of dis- tance education to the engineering community.

\section{DEPLOYMENT OF REMOTE EXPERI- MENTS}

The main concept in turning a locally-controlled experiment into a remotely-controlled one consists of moving the user interface of the monitoring and control software away from the physical system. Such a paradigm enables a flexible access that is valuable for teachers looking for live classroom demonstration facilities or for students wishing or having to carry out real-world laboratory projects to sustain their learning activities.

In automatic control education, different experiments have to support different concepts taught in control courses like controller tuning, model development, and the actual programming and implementation of a control scheme or algorithm. A rich variety of control experiments can be carried out remotely in the ReLAX framework. Three different laboratory experiments will initially be available through the Cyberlab network; one at EPFL, NTNU and RUB, respectively. Some typical examples from these experiments are:

\section{Experiments where the user acts as an operator for the laboratory process.}

Typically, the user will change the reference values of some control loops and monitor the equipment. Further, the user may change some control parameter settings, such as the gain and integral time of a PI-controller. Such parameter changes may be based on intuition only, or result from a systematic tuning procedure, which may be supported in the experimentation environment. The experiment may also be designed to introduce disturbances in order to validate the dynamic performances obtained with the user's settings. In Fig. 2 an example of a user inter-

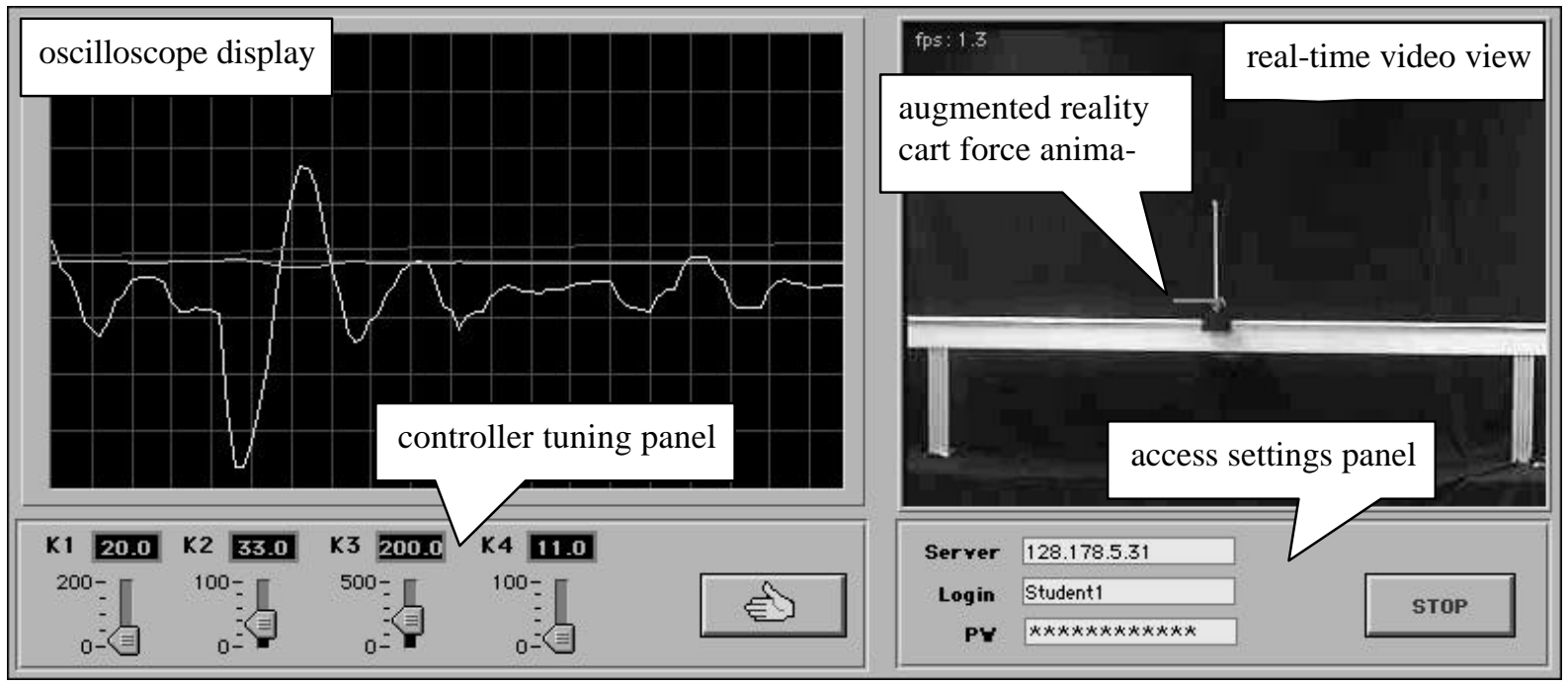

Fig. 2. Screenshot of the inverted pendulum client software. 
face for making these kinds of experiments with an inverted pendulum is shown (Gillet et al., 2000).

This pendulum emulates in two dimensions a juggler trying to keep a broomstick in equilibrium on his fingertip. The control objective is to simultaneously keep the pendulum stick in the upright position and the supporting cart at the centre of the rail, using a single actuator.

The inverted pendulum is made up of a 4-meter horizontal rail mounted on top of a case that contains both the electronics and the power supply. A cart, moved by an electric motor by means of a metallic belt, can slide along this rail. The rotational axis of the pendulum stick is on this cart. No actuator exists for this axle. The only way to change the stick position is to accelerate the cart. Hardware security switches that turn the power off can be found at each end of the rail.

Any type of computer equipped with a National Instruments PCI-1200 family DAQ board can control this laboratory-scale system. In the actual implementation, the computer is an Apple Power Macintosh G3 which handling simultaneously the realtime control, the video processing and broadcasting, the transactions with the service provider, as well as the data exchange with remote users.

The control software piloting the real process locally is a virtual instrument (VI) built using LabVIEW. An in-house real-time kernel extends the LabVIEW capability and guarantees high speed and accurate pace. The VI provides a complete interface between the remote users and the real process. It is used to generate excitation signals and observe corresponding responses.

The control software has a fixed state-feedback structure. This multivariable controller aims at minimising the energy spent by the actuator to keep the stick raised and the cart at the centre of the rail. The implementation relies on both angular and longitudinal position measurements, as well as the respective velocity estimates. For security reasons, the tuning options are restricted to changes of the controller gains.

\section{Experiments in which the user may learn about in- dustrial control systems, including instrumentation and actuators}

Learning about industrial control systems, including instrumentation and actuators is performed by activating hyperlinks on a Process and Instrument Diagram of the Remote Laboratory. A link may e.g. point to a datasheet for a valve. The refrigeration process located at NTNU is suited for this since it contains a number of different instruments, valves and process elements. This process itself consists of a compressor, condenser, expansion valve, and an evaporator, as shown schematically on the left-hand side of Fig. 3. The process will cool down the liquid in a tank. The fluid in the tank is heated. This is the main process disturbance.

The process is equipped with approximately 30 tem-

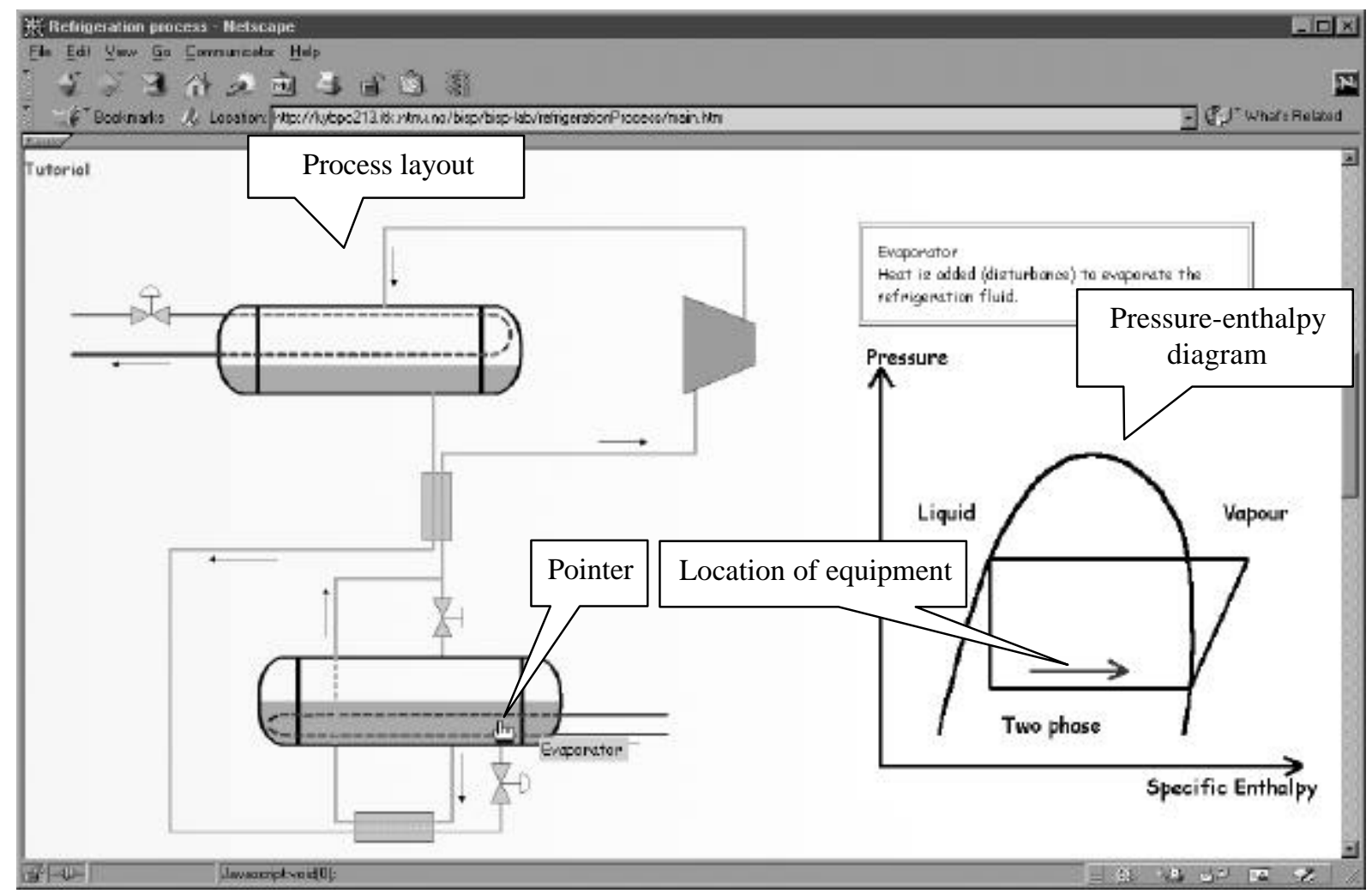

Fig. 3. Tutorial material on the process is integrated with drawings showing the process layout. 
perature, pressure, level and flow transmitters, and 10 valves. The instrumentation (not shown in Fig. 3) is connected to a Simatic S7-400 PLC, which again is connected to an industry standard PC. This PC has software for communicating with the PLC and for programming the PLC. In addition, Simatic WinCC is installed on the PC, and is used both for a local HMI and for communicating with a Web server through an OPC interface. The Web server uses the APIS program suite from Prediktor AS to access process data from WinCC and make this data available over the Web.

The use of Web technology also allows for a tight integration of tutorial material on the process or the control theory, which the experiments are designed to demonstrate. This is illustrated in Fig. 3, where the screenshot of the Web page shows the process layout, see also Foss et al., 2000. By pointing to key equipment in the process, a pressure-enthalpy diagram pops up on the right-hand side, in which a refrigeration cycle is drawn in. It also explains where the equipment pointed to is located in the pressureenthalpy diagram.

The user may run different experiments on the refrigeration cycle.

- The user may run identification experiments by perturbing the system and collecting process data. These data may be used to identify an empirical dynamical model, to determine parameters in model of the process, or to validate a model. Both open-loop and closed-loop experiments may be run.

- The user may test different pre-programmed control structures. The refrigeration process is particularly suited for testing different control structures.

- The user may program a controller and download it to the control system, and then assess its performance by running experiments with this controller. This opens possibilities both for giving students more realistic experience with advanced control concepts, as well as to provide researchers with a test facility for new controller algorithms that is closer to industrial reality than what simulation alone can offer.

\section{Experiments with a feeling of being present in the laboratory}

One important issue that needs to be addressed in remote experimentation is how to give the user the necessary feeling of interacting with real, physical equipment. The ability to manipulate the experiment remotely, and seeing the results of such manipula-

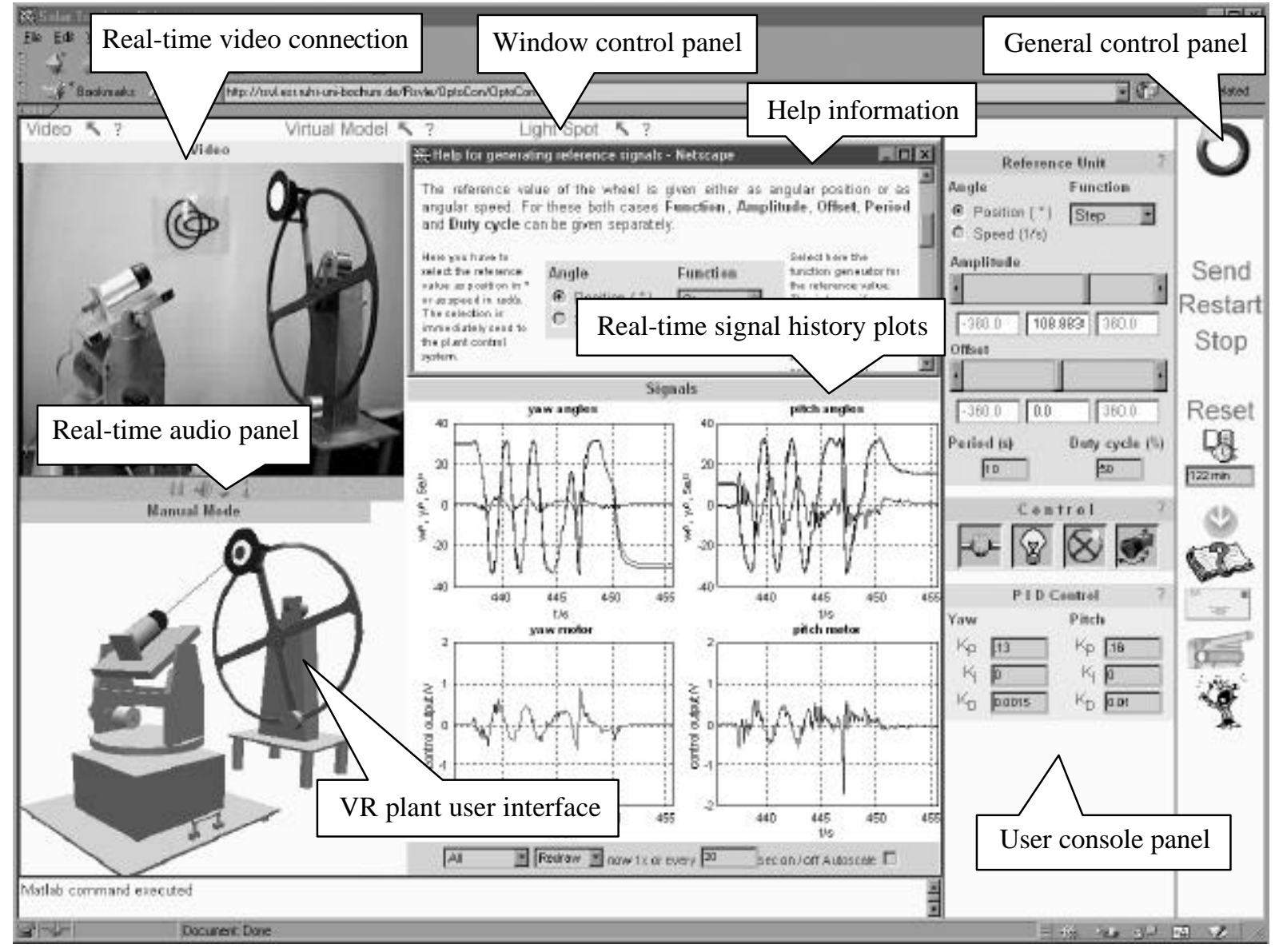

Fig. 4. Screenshot example of the GUI for the optical tracking plant with virtual-reality plant user interface 
tions in real time, will contribute to this aim, see Fig. 2. The feeling of being present at the experimental site can be further enhanced by the transmission of sound and video images from the experiment, and by the use of virtual-reality techniques. Fig. 4 shows an example from an optical tracking plant (Junge and Schmid, 2000), where the experiment can be optionally controlled by a virtual-reality (VR) model and the reaction of the plant can be immediately observed by the video and audio connection.

The plant is composed of two units. The reference unit includes a light source fixed on a wheel. A motor moving the light spot on a circular trajectory drives this wheel. The user can select the angle (i.e. position of the light spot) or the angular velocity of the wheel (i.e. speed of the light spot). It is also possible to change the properties of the signal generator (e.g. shape, amplitude, offset, period) attained to the angle or to the angular velocity. The tracking unit is composed of a tracking sensor mounted on a $2 \mathrm{DOF}$ support, which can be rotated vertically and laterally, necessary to track the light spot. Two DC motors, each of which is used to control the rotation, drive this support. The tracking sensor is build according to an insect eye including a matrix of phototransistors to detect vertical and horizontal deviations of the light spot from the optical centre. The vertical deviation is fed to a controller for yaw angle control, while the horizontal deviation is fed to a second controller for pitch angle control. Both controllers are used for tracking the light spot.

The maximum angular velocity of the wheel is 2 $\mathrm{rad} / \mathrm{sec}$ and the corresponding maximum vertical and lateral angle turns of the support for tracking the spot on the circle are $\pm 36^{\circ}$. As the tracking camera has a limitation in the view angle of $\pm 12^{\circ}$, it is a challenge for students to design a controller such that the light spot will not leave the camera's view.

Details about the modular structure of the remote laboratory environment of this experiment based on MATLAB/SIMULINK is described by Schmid, 2001.

The Web page necessary to control this experiment is organised in the form of a cockpit, Fig. 4. The main operating actions (e.g. start/stop an experiment session, or reset the plant to a predefined initial state) are located in the general control panel frame. The specific commands to operate the plant as well as parameters to modify the characteristics of the reference signal and of the implemented controller are found in the user console panel. Although the lack of a direct physical contact with the experiment may lead to a loss of 'practical feeling', it can be compensated by enhanced multimedia components. An VR plant user interface enables the user to operate the real plant manually by mouse actions in the virtual reality scene of the 3-D model. The actions performed with this model are immediately converted into commands and transferred to the experimental site. Although the user can inspect the plant by the video camera or tune the controller by listening to the sound the plant produces, a graphical representation of measured signals is needed to show small variations and to generate a report.

\section{CONCLUSIONS}

This paper describes a model for remote experimentation including three parties: customers or users, providers of experiments and an organising company acting as an Experiment Service Provider. Further, the paper shows how a diversified set of high-quality experiments can be made available on the Internet, and how these can be designed to facilitate the learning process of engineering students. The described Cyberlab network effectively enables the establishment of a distributed laboratory for hands-on automatic control education at the European level. The sharing of experiments offers a unique set of didactic resources for students in engineering.

\section{REFERENCES}

Antsaklis, P., T. Basar, R. De Carlo, N.H. McClamroch, M. Spong and S. Yurkovich (1999). Report on the NSF/CSS Workshop on New Directions in Control Engineering Education. IEEE Control Systems Magazine, 19, No. 5, pp. 53-58.

Foss, B.A., T.I. Eikaas and M. Hovd (2000). Merging physical experiments back to the learning arena. Proc. American Control Conference ACC'2000, Chicago, pp. 2944-2948.

Gillet, D., C. Salzmann, H.A. Latchman and O.D. Crisalle (2000). Recent Advances in Remote Experimentation. Proc. American Control Conference ACC'2000, Chicago, pp. 2955-2956.

Junge, Th.F. and Chr. Schmid (2000). Web-Based Remote Experimentation Using a LaboratoryScale Optical Tracker. Proc. American Control Conference ACC'2000, Chicago, pp. 2951-2954.

Kheir N.A., K.J. Åström, D. Auslander, K.C. Cheok, G.F. Franklin, M. Masten and M. Rabins (1996). Control Systems Engineering Education. Automatica, 32 , No. 2, pp. 147-166.

ReLAX (2001). IST project IST-1999-20827. Web page http://www.relax-project.com

Schmid, Chr. (2001). Web-Based Remote Experimentation. Proc IFAC Conference on Telematics Applications in Automation and Robotics, Weingarten.

\section{Acknowledgement}

This work was supported by the EU IST Programme under project contract IST-1999-20827. 